\title{
Closed-form expression for mutual intensity evolution of Hermite-Laguerre-Gaussian Schell-model beams
}

\author{
Eugeny Abramochkin ${ }^{1}$ and Tatiana Alieva ${ }^{2, *}$ \\ ${ }^{1}$ Lebedev Physical Institute, Samara, 443011, Russia \\ ${ }^{2}$ Universidad Complutense de Madrid, Facultad de Ciencias Físicas, Ciudad Universitaria s/n, Madrid 28040, Spain \\ *Corresponding author: talieva@fis.ucm.es
}

Compiled September 5, 2017

\begin{abstract}
We derive a comprehensive closed-form expression for the evolution of the mutual intensity (MI) of HermiteLaguerre-Gaussian Schell-model beams (HLG-SMBs) during propagation through rotationally symmetric optical systems. We demonstrate that the MI of the beam associated with a given HLG mode at any transverse plane can be presented as a linear superposition of the MIs of the SMBs associated with the equal and lower index modes of the same type but of complex argument. The obtained expression allows easily analyzing the evolution of the intensity distribution and the crosscorrelation function of such beams and, in particular, understanding the coherence singularity formation and modification during the beam propagation. (๑) 2017 Optical Society of America
\end{abstract}

OCIS codes: (030.0030) Coherence and statistical optics; (260.6042) Singular optics; (070.2575) Fractional Fourier transforms

http://dx.doi.org/10.1364/ol.XX.XXXXXX

Structurally stable beams which propagate in rotationally symmetric optical systems without changing their complex field amplitude except of possible scaling, rotation and additional quadratic phase are exploiting for different applications: optical communications, micro and nano-particle manipulation, imaging, metrology, etc. [1, 2]. In particular, their easily recognized intensity patterns and phase singularities make them attractive for information encoding [2,3]. However, the deformations during propagation through random medium and speckle noise typical for coherent beams have waked up the interest to their partially coherent counterparts which have been found more robust [4]. Analytic description of scalar monochromatic partially coherent light propagation in paraxial approximation requires application of bilinear functions (for example, mutual intensity (MI), Wigner distribution (WD), and ambiguity function (AF)) that significantly increases the problem complexity. Thus, the MI is defined as $\Gamma\left(\mathbf{r}_{1}, \mathbf{r}_{2}\right)=\left\langle f\left(\mathbf{r}_{1}\right) f^{*}\left(\mathbf{r}_{2}\right)\right\rangle$ [5], where $f(\cdot)$ is a complex field amplitude, $\mathbf{r}=(x, y)^{t}$ is a position vector in the plane transverse to the beam propagation direction $z$ and the brackets $\langle\cdot\rangle$ stand for ensemble averaging which is amended for the coherent case. In particular,
$\Gamma(\mathbf{r}, \mathbf{r})=\left\langle|f(\mathbf{r})|^{2}\right\rangle=I(\mathbf{r})$ corresponds to the beam intensity distribution while $\Gamma(\mathbf{r},-\mathbf{r})=C(\mathbf{r})$ is known as cross-correlation function (CCF) which is often applied for the correlation analysis of symmetrical beams [6-9]. Schell model is probably the most used one for the description of the partially coherent beams since the experimental conditions for its applicability are often satisfied. In this case the MI expressed in the coordinates $\mathbf{R}=(X, Y)^{t}=\frac{1}{2}\left(\mathbf{r}_{1}+\mathbf{r}_{2}\right)$ and $\mathbf{r}=\mathbf{r}_{1}-\mathbf{r}_{2}$ is given in the simplified form in the input plane $z=0$ as

$$
\Gamma\left(\mathbf{R}+\frac{\mathbf{r}}{2}, \mathbf{R}-\frac{\mathbf{r}}{2}\right)=f_{\mathcal{c}}\left(\mathbf{R}+\frac{\mathbf{r}}{2}\right) f_{\mathcal{c}}^{*}\left(\mathbf{R}-\frac{\mathbf{r}}{2}\right) \gamma(\mathbf{r})
$$

where $\gamma(\mathbf{r})$ is a degree of spatial coherence (DoC) and $f_{c}(\mathbf{r})$ is a complex field amplitude of an associated coherent beam. Note that the Schell model beam (SMB) can be considered as a stochastic generalization of the coherent one and often heritages some of its properties [10]. Further it is assumed that the DoC has a typical Gaussian form $\gamma(\mathbf{r})=\exp \left(-\frac{1}{2} \pi \sigma|\mathbf{r}|^{2}\right)$. We observe that the MI of a SMB is described as a product of the shift-invariant $\mathrm{DoC}$ and the MI corresponding to coherent beam. However, this simple relation is only valid in the input plane $z=0$ and is destroyed during beam propagation. The analysis of field correlation and the intensity distributions in other planes becomes difficult even in the case when $f_{c}$ is a structurally stable beam.

Here we derive a closed-form expression for the transport of the MI through rotationally symmetric optical system for a wide class of the SMBs which are associated with structurally stable coherent Hermite-Laguerre-Gaussian (HLG) modes [1, 11]. We recall that coherent HLG beams include HermiteGaussian (HG) modes and well-known optical vortices - helical Laguerre-Gaussian (LG) modes. The goal of this Letter is to study the evolution of their partially coherent counterpart. While this problem in particular cases of HG-SMBs and LGSMBs has been considered in Refs. [12] and [13], correspondingly, the results reported here apart from covering more general case provide a comprehensive closed-form expression for the MI evolution. We demonstrate that the MI of a HLG-SMB in any transverse plane can be presented as a linear superposition of the MIs of the SMBs associated with the equal and lower index HLG modes of the same type but of complex argument. The obtained expression can be considered as an alternative to the coherent mode decomposition of the MI [14] (see, 
for example, HG mode expansion of the MI for a Gaussian SMB [15]) in the sense that it allows easily analyzing the evolution of the MI and therefore the beam intensity and correlation during beam propagation. In particular, the result provides a theoretical background for the study of the birth and evolution of the coherence singularities found in Refs. [6-9].

Here the orthonormal HLG modes are defined as [16]

$$
\mathrm{HLG}_{n, m}(\mathbf{r} \mid \beta)=c_{n, m} \exp \left(-\pi|\mathbf{r}|^{2}\right) H L_{n, m}(\mathbf{r} \mid \beta),
$$

where $c_{n, m}=1 / \sqrt{2^{n+m-1} n ! m !}$ are the normalization constants and $H L_{n, m}(\mathbf{r} \mid \beta)$ are Hermite-Laguerre (HL) polynomials obtained from the generating function $G(\mathbf{r}, \mathbf{s} \mid \beta)$ as

$$
H L_{n, m}\left((\mathbf{r} \mid \beta)=\left.\partial_{s_{x}}^{n} \partial_{s_{y}}^{m} G(\mathbf{r}, \mathbf{s} \mid \beta)\right|_{\mathbf{s}=\mathbf{0}} .\right.
$$

The generating function which will be a protoganist in the later calculations is given by

$$
\begin{aligned}
G(\mathbf{r}, \mathbf{s} \mid \beta) & =\sum_{n=0}^{\infty} \sum_{m=0}^{\infty} H L_{n, m}(\mathbf{r} \mid \beta) \frac{s_{x}^{n} s_{y}^{m}}{n ! m !} \\
& =\exp \left[-\left(\mathbf{U}^{*} \mathbf{s}\right)^{t}(\mathbf{U s})+2 \sqrt{2 \pi}\left(\mathbf{U}^{*} \mathbf{s}\right)^{t} \mathbf{r}\right],
\end{aligned}
$$

where $\mathbf{s}=\left(s_{x}, s_{y}\right)^{t}$ and $\mathbf{U}=\left(\begin{array}{c}\cos \beta i \sin \beta \\ i \sin \beta \cos \beta\end{array}\right)$.

For $\beta=0$ and $\beta=\pi / 4$ HLG modes are reduced to HG and LG modes, correspondingly. In the later case the usually used azimuthal and radial indices of the LG mode are given by $\ell=n-m$ and $p=\min (n, m)$. Note also that $H L_{n, m}(-\mathbf{r} \mid \beta)=$ $(-1)^{n+m} H L_{n, m}(\mathbf{r} \mid \beta)$. Here and later, we use dimensionless variables: the position vector is normalized on the effective size of the fundamental Gaussian mode $w, \mathbf{r} \rightarrow \mathbf{r} / w$.

A beam propagation through a paraxial rotationally symmetric optical system is described by the canonical integral transform defined by three free parameters $g, \lambda$ and $\alpha$ often gathered in a scalar $2 \times 2$ ray transformation matrix $\mathbf{T}=\left(\begin{array}{ll}a & b \\ c & d\end{array}\right)$ with $\operatorname{det} \mathbf{T}=1$, where $g=(a c+b d) /\left(a^{2}+b^{2}\right), \lambda=\sqrt{a^{2}+b^{2}}$, and $\alpha=\arctan (b / a)$. Here again the matrix elements are dimensionless that means $b \rightarrow b / w^{2}$ and $c \rightarrow w^{2} c$. Using the modified Iwasawa decomposition it was shown that the matrix $\mathbf{T}_{\alpha}=\left(\begin{array}{cc}\cos \alpha & \sin \alpha \\ -\sin \alpha & \cos \alpha\end{array}\right)$ corresponding to the fractional Fourier transform (FrFT) is responsible for structural beam changes, while the parameters $\lambda$ and $g$ lead to scaling and quadratic phase modulation, respectively (see, for example, Ref. [1]). This fact is reflected in the following relation for the MI in the output of the system described by $\mathbf{T}$ :

$$
\begin{aligned}
& \Gamma\left(\mathbf{R}+\frac{\mathbf{r}}{2}, \mathbf{R}-\frac{\mathbf{r}}{2} \mid \mathbf{T}\right) \\
& =\frac{1}{\lambda^{2}} \exp \left(i 2 \pi g \mathbf{R}^{t} \mathbf{r}\right) \Gamma\left[\frac{1}{\lambda}\left(\mathbf{R}+\frac{\mathbf{r}}{2}\right), \frac{1}{\lambda}\left(\mathbf{R}-\frac{\mathbf{r}}{2}\right) \mid \mathbf{T}_{\alpha}\right],
\end{aligned}
$$

that reduces the problem to the search of the $\Gamma\left(\mathbf{R}+\frac{1}{2} \mathbf{r}, \mathbf{R}-\right.$ $\left.\frac{1}{2} \mathbf{r} \mid \mathbf{T}_{\alpha}\right)$. We recall that for the propagation in the homogeneous medim $\alpha=0$ corresponds to the input plane, while $\alpha=\pi / 2$ describes the far field. It has been shown [9] that the evolution of the SMBs associated with structurally stable beams during propagation through a paraxial optical system can be easier understood using the ambiguity function given by

$$
A(\mathbf{r}, \mathbf{p})=\int \Gamma\left(\mathbf{R}+\frac{\mathbf{r}}{2}, \mathbf{R}-\frac{\mathbf{r}}{2}\right) \exp \left(-i 2 \pi \mathbf{R}^{t} \mathbf{p}\right) \mathrm{d} \mathbf{R},
$$

where $\mathbf{p}=\left(p_{x}, p_{y}\right)^{t}$ is a dimensionless spatial frequency vector $\mathbf{p} \rightarrow w \mathbf{p}$. Indeed, taken into account 1) that the FrFT produces rotation of the AF (see, for example, Ref. [17])

$$
A\left(\mathbf{r}, \mathbf{p} \mid \mathbf{T}_{\alpha}\right)=A\left(\mathbf{r} \cos \alpha-\mathbf{p} \sin \alpha, \mathbf{r} \sin \alpha+\mathbf{p} \cos \alpha \mid \mathbf{T}_{0}\right),
$$

where $\mathbf{T}_{0}=\mathbf{I}$ is a unity matrix indicated the input plane, 2) that the HLG modes, $\mathrm{HLG}_{n, m}(\mathbf{r} \mid \beta)$, are eigenfunctions of the FrFT and then their AFs do not change under such phase space rotation: $A_{n, m, \beta}^{(0)}\left(\mathbf{r}, \mathbf{p} \mid \mathbf{T}_{\alpha}\right)=A_{n, m, \beta}^{(0)}\left(\mathbf{r}, \mathbf{p} \mid \mathbf{T}_{0}\right)$ and 3) Eq. (1), we can invert Eq. (6) and write the MI as

$$
\begin{aligned}
\Gamma_{n, m, \beta}^{(\sigma)} & \left(\mathbf{R}+\frac{\mathbf{r}}{2}, \mathbf{R}-\frac{\mathbf{r}}{2} \mid \mathbf{T}_{\alpha}\right)=\int A_{n, m, \beta}^{(0)}(\mathbf{r}, \mathbf{p}) \\
& \times \exp \left(-\frac{\pi \sigma}{2}|\mathbf{r} \cos \alpha-\mathbf{p} \sin \alpha|^{2}\right) \exp \left(i 2 \pi \mathbf{R}^{t} \mathbf{p}\right) \mathrm{d} \mathbf{p} .
\end{aligned}
$$

Here and later, in the notation of the MI, AF, CCF, WD, and intensity functions we use subindices $n, m$, and $\beta$ to indicate the associated HLG mode, while superindex $\sigma$ defines the coherence state of the HLG-SMB. In particular, superindex 0 corresponds to the coherent case.

Moreover, the expression for the AF of the HLG mode can be easily obtained from the known one corresponding to the Wigner distribution function $W$ [18], since $W_{n, m, \beta}^{(0)}(\mathbf{r}, \mathbf{p})=$ $4(-1)^{n+m} A_{n, m, \beta}^{(0)}(2 \mathbf{r}, 2 \mathbf{p})$ that follows from the symmetry of the HL polynomials. Thus,

$$
A_{n, m, \beta}^{(0)}(\mathbf{r}, \mathbf{p})=A_{0,0, \beta}^{(0)}(\mathbf{r}, \mathbf{p}) L_{n}\left(\pi\left|\mathbf{R}_{1}\right|^{2}\right) L_{m}\left(\pi\left|\mathbf{R}_{2}\right|^{2}\right)
$$

with $A_{0,0, \beta}^{(0)}(\mathbf{r}, \mathbf{p})=\exp \left(-\frac{1}{2} \pi|\mathbf{r}|^{2}-\frac{1}{2} \pi|\mathbf{p}|^{2}\right)$ and

$$
\begin{aligned}
& \mathbf{R}_{1}=\left(x \cos \beta+p_{y} \sin \beta, y \sin \beta-p_{x} \cos \beta\right)^{t}, \\
& \mathbf{R}_{2}=\left(x \sin \beta-p_{y} \cos \beta, y \cos \beta+p_{x} \sin \beta\right)^{t} .
\end{aligned}
$$

In order to calculate the integral Eq. (8) the generating funtion approach is used. We have noticed that $H L_{n, n}(\mathbf{r} \mid \pi / 4)=$ $(-2 i)^{n} n ! L_{n}\left(2 \pi|\mathbf{r}|^{2}\right)$ and that the generating function of the HL polynomials (see Eq.(4)) has an exponential form with second order complex polynomial. Then, expressing the $A_{n, m, \beta}^{(0)}(\mathbf{r}, \mathbf{p})$ through the generating function the integral in Eq. (8) can be evaluated and the MI can be found by proper derivation of the result. For this purpose we introduce an auxiliary function:

$$
\begin{aligned}
& \mathfrak{A}_{n, m, N, M}(\mathbf{r}, \mathbf{p}) \\
& =A_{0,0, \beta}^{(0)}(\mathbf{r}, \mathbf{p}) H L_{n, N}\left(\frac{\mathbf{R}_{1}}{\sqrt{2}} \mid \frac{\pi}{4}\right) H L_{m, M}\left(\frac{\mathbf{R}_{2}}{\sqrt{2}} \mid \frac{\pi}{4}\right),
\end{aligned}
$$

which is reduced to $A_{n, m, \beta}^{(0)}(\mathbf{r}, \mathbf{p})$ for $N=n$ and $M=m$. Then we can express the generating function of $\mathfrak{A}_{n, m, N, M}(\mathbf{r}, \mathbf{p})$ in the exponential form as

$$
\begin{aligned}
\mathfrak{G}(\mathbf{r}, \mathbf{p}, \mathbf{s}, \mathbf{t}) & =\sum_{n, N=0}^{\infty} \sum_{m, M=0}^{\infty} \mathfrak{A}_{n, m, N, M}(\mathbf{r}, \mathbf{p}) \frac{s_{x}^{n} s_{y}^{N}}{n ! N !} \frac{t_{x}^{m} t_{y}^{M}}{m ! M !} \\
& =A_{0,0, \beta}^{(0)}(\mathbf{r}, \mathbf{p}) G\left(\frac{\mathbf{R}_{1}}{\sqrt{2}, \mathbf{s}} \mid \frac{\pi}{4}\right) G\left(\frac{\mathbf{R}_{2}}{\sqrt{2}}, \mathbf{t} \mid \frac{\pi}{4}\right) .
\end{aligned}
$$

Substituting the generating function $\mathfrak{G}(\mathbf{r}, \mathbf{p}, \mathbf{s}, \mathbf{t})$ in Eq. (8):

$$
\begin{aligned}
\Gamma_{n, m, \beta}^{(\sigma)} & \left(\mathbf{R}+\frac{\mathbf{r}}{2}, \mathbf{R}-\frac{\mathbf{r}}{2} \mid \mathbf{T}_{\alpha}\right)=\frac{i^{n+m}}{2^{n+m} n ! m !} \\
& \times \partial_{s_{x}}^{n} \partial_{t_{x}}^{m} \partial_{s_{y}}^{n} \partial_{t_{y}}^{m}\left\{\int \exp \left(-\frac{\pi \sigma}{2}|\mathbf{r} \cos \alpha-\mathbf{p} \sin \alpha|^{2}\right)\right. \\
& \left.\times \exp \left(i 2 \pi \mathbf{R}^{t} \mathbf{p}\right) \mathfrak{G}(\mathbf{r}, \mathbf{p}, \mathbf{s}, \mathbf{t}) \mathrm{d} \mathbf{p}\right\}\left.\right|_{\mathbf{s}=\mathbf{t}=\mathbf{0}}
\end{aligned}
$$

we evaluate the integral in braces applying the formula [16], $\int \exp \left(-\pi \mathbf{p}^{t} \mathbf{M} \mathbf{p}-i 2 \pi \mathbf{p}^{t} \mathbf{q}\right) \mathrm{d} \mathbf{p}=\frac{1}{\sqrt{\operatorname{det} \mathbf{M}}} \exp \left(-\pi \mathbf{q}^{t} \mathbf{M}^{-1} \mathbf{q}\right)$, 
where $\mathbf{M}$ is a symmetric matrix whose real part is positive definite. The result again is an exponential with second order complex polynomials. It can be expressed, similarly to Eq. (12), as a product of generating functions of the HL polynomials, Eq. (4), but of complex valued arguments and parameter $\beta$. Then after the differentiation and tedious but straightforward calculus we derive the expression for the MI of the HLG-SMB

$$
\begin{aligned}
& \Gamma_{n, m, \beta}^{(\sigma)}\left(\mathbf{R}+\frac{\mathbf{r}}{2}, \mathbf{R}-\frac{\mathbf{r}}{2} \mid \mathbf{T}_{\alpha}\right)=\frac{1}{\left(1+\sigma \sin ^{2} \alpha\right)^{n+m+1}} \\
& \times \exp \left(-\frac{2 \pi|\mathbf{R}|^{2}-i \pi \sigma \sin 2 \alpha \mathbf{R}^{t} \mathbf{r}+\frac{1}{2} \pi(1+\sigma)|\mathbf{r}|^{2}}{1+\sigma \sin ^{2} \alpha}\right) \\
& \times \sum_{v=0}^{n} \sum_{\mu=0}^{m}\left(\begin{array}{c}
n \\
v
\end{array}\right)\left(\begin{array}{c}
m \\
\mu
\end{array}\right) c_{v, \mu}^{2}\left(\sigma \sin ^{2} \alpha\right)^{n+m-v-\mu} \\
& \times H L_{v, \mu}\left(\frac{\mathbf{R}+\frac{1}{2} q \mathbf{r}}{\sqrt{1+\sigma \sin ^{2} \alpha}} \mid \beta\right) H L_{v, \mu}^{*}\left(\frac{\mathbf{R}-\frac{1}{2} q \mathbf{r}}{\sqrt{1+\sigma \sin ^{2} \alpha}} \mid \beta\right),
\end{aligned}
$$

where $q=1+\sigma \sin ^{2} \alpha-i \sigma \sin \alpha \cos \alpha$ is an auxiliary complex parameter used for brevity. Eq. (14) can also be rewritten as

$$
\begin{aligned}
& \Gamma_{n, m, \beta}^{(\sigma)}\left(\mathbf{R}+\frac{\mathbf{r}}{2}, \mathbf{R}-\frac{\mathbf{r}}{2} \mid \mathbf{T}_{\alpha}\right)=\frac{1}{\left(1+\sigma \sin ^{2} \alpha\right)^{n+m+1}} \\
& \times \exp \left(-\frac{\pi \sigma}{2}|\mathbf{r}|^{2} \cos 2 \alpha\right) \sum_{v=0}^{n} \sum_{\mu=0}^{m}\left(\begin{array}{l}
n \\
v
\end{array}\right)\left(\begin{array}{l}
m \\
\mu
\end{array}\right)\left(\sigma \sin ^{2} \alpha\right)^{n+m-v-\mu} \\
& \times \Gamma_{v, \mu, \beta}^{(0)}\left(\frac{\mathbf{R}+\frac{1}{2} q \mathbf{r}}{\sqrt{1+\sigma \sin ^{2} \alpha}}, \frac{\mathbf{R}-\frac{1}{2} q \mathbf{r}}{\sqrt{1+\sigma \sin ^{2} \alpha}} \mid \mathbf{T}_{0}\right)
\end{aligned}
$$

where for compactness we introduced the notation for the MI of the HLG modes allowing complex position variables

$$
\Gamma_{v, \mu, \beta}^{(0)}\left(\mathbf{r}_{1}, \mathbf{r}_{2} \mid \mathbf{T}_{0}\right)=\mathrm{HLG}_{v, \mu}\left(\mathbf{r}_{1} \mid \beta\right) \mathrm{HLG}_{v, \mu}^{*}\left(\mathbf{r}_{2} \mid \beta\right) .
$$

Note that it is a nonnegative definite Hermitian function of variables $\mathbf{r}_{1}$ and $\mathbf{r}_{2}$. The closed-form expressions Eq. (14) and Eq. (15) for the evolution of the MI of the HLG-SMB are a principal result of the paper. It is easy to see that the MI of a HLGSMB with indices $n$ and $m$ is transformed apart from Gaussian multiplicative term to a linear superposition of the MIs of the HLG modes of equal and lower indices of the same type (parameter $\beta$ is preserved) but of complex argument. The weights of the modes depend on the degree of the coherence (parameter $\sigma$ ) and the propagation distance (parameter $\alpha$ ). The weight of the lower modes is increased during propagation and is larger for lower coherence SMB. As it is expected in the coherent case, $\sigma=0$ and therefore $q=1$, the MI of any mode does not change. Note that the arguments of the modes are real in the input plane $(\alpha=0)$ and in the far field $(\alpha=\pi / 2)$.

We observe that the intensity distribution $I_{n, m, \beta}^{(\sigma)}\left(\mathbf{R} \mid \mathbf{T}_{\alpha}\right)$ obtained from Eq. (14) or Eq. (15) for $\mathbf{r}=\mathbf{0}$,

$$
\begin{aligned}
& I_{n, m, \beta}^{(\sigma)}\left(\mathbf{R} \mid \mathbf{T}_{\alpha}\right)=\frac{1}{\left(1+\sigma \sin ^{2} \alpha\right)^{n+m+1}} \sum_{\nu=0}^{n} \sum_{\mu=0}^{m}\left(\begin{array}{l}
n \\
v
\end{array}\right)\left(\begin{array}{l}
m \\
\mu
\end{array}\right) \\
& \times\left(\sigma \sin ^{2} \alpha\right)^{n+m-v-\mu} I_{v, \mu, \beta}^{(0)}\left(\frac{\mathbf{R}}{\sqrt{1+\sigma \sin ^{2} \alpha}} \mid \mathbf{T}_{0}\right),
\end{aligned}
$$

is expressed as a superposition of the intensity distributions of the HLG modes of equal and lower indices. It does not have zeros except of the input plane corresponding to $\alpha=0$ where only one mode is present in the sum and $I_{n, m, \beta}^{(\sigma)}\left(\mathbf{R} \mid \mathbf{T}_{0}\right)=$ $I_{n, m, \beta}^{(0)}\left(\mathbf{R} \mid \mathbf{T}_{0}\right)=\left|\mathrm{HLG}_{n, m}(\mathbf{R} \mid \beta)\right|^{2}$, because the roots of the HL polynomials never coincide.
On the other hand, for $\mathbf{R}=\mathbf{0}$ Eq. (14) and Eq. (15) are reduced to the CCF:

$$
\begin{aligned}
& C_{n, m, \beta}^{(\sigma)}\left(\mathbf{r} \mid \mathbf{T}_{\alpha}\right)=\frac{1}{\left(1+\sigma \sin ^{2} \alpha\right)^{n+m+1}} \\
& \times \exp \left(-\frac{2 \pi(1+\sigma)}{1+\sigma \sin ^{2} \alpha}|\mathbf{r}|^{2}\right) \sum_{v=0}^{n} \sum_{\mu=0}^{m}(-1)^{v+\mu}\left(\begin{array}{l}
n \\
v
\end{array}\right)\left(\begin{array}{c}
m \\
\mu
\end{array}\right) \\
& \times c_{v, \mu}^{2}\left(\sigma \sin ^{2} \alpha\right)^{n+m-v-\mu}\left|H L_{v, \mu}\left(\frac{q \mathbf{r}}{\sqrt{1+\sigma \sin ^{2} \alpha}} \mid \beta\right)\right|^{2} .
\end{aligned}
$$

Here the polynomial symmetry has been used again. It is easy to see that zeros of the CCF in the input plane coincide with the intensity zeros: $C_{n, m, \beta}^{(\sigma)}\left(\mathbf{r} \mid \mathbf{T}_{0}\right)=(-1)^{n+m} \gamma(2 \mathbf{r}) I_{n, m, \beta}^{(0)}\left(\mathbf{r} \mid \mathbf{T}_{0}\right)$. The expression Eq. (18) is useful, in particular, for the study of the birth and evolution of the coherence singularities [19, 20]. The analysis of the coherence singularities is often related to the study of the zero curves of the CCF, which as we see from Eq. (18) is reduced to the search of the real roots of the 2D polynomial of order $2(n+m)$ written as a sum in this equation. Even for the same HLG mode the form of the root (and therefore singularity) curves changes depending on the parameters $\alpha$ and $\sigma$. From the symmetry of the HG and LG modes it follows that singularity curves are lines and rings, correspondingly. It verifies the semi-empiric results obtained in Refs. [6-8] where the phase singularities of the LG-SMB CCF in far field have been considered. The birth and evolution of the CCF singularities have been studied in Ref. [9] on the example of low-order HG-SMBs. The derived expression Eq. (18) is suitable for more deep analysis of such singularities in the general case of HLGSMBs of any order. Note that the robustness of the coherence singularities might be use for information codification in the similar manner it was done for the case of the coherent modes. We underline that a simple interferometric setup $[7,8]$ allows measuring the CCF.

As an example the evolution of the intensity and CCF during beam propagation for the HLG-SMBs of indices $n=3$ and $m=1$ for $\beta=0$ (HG-SMB), $\beta=\pi / 8$ (HLG-SMB), and $\beta=\pi / 4$ (LG-SMB) is considered in Fig. 1. The intensity and the CCF distributions in three transverse planes corresponding to $\alpha=0$ (input plane), $\alpha=\pi / 4$ (Fresnel region), and $\alpha=\pi / 2$ (far field) for three different values of coherence parameter: $\sigma=0.25, \sigma=1$, and $\sigma=10$ are displayed. Note that the coherence radius is related to the waist of the fundamental Gaussian mode as $w \sqrt{\sigma}$. It is easy to see that the position, the numbers and, in the case $\beta=\pi / 8$, the form of the singularity curves are changed during beam propagation. As it follows from Eq. (18) the maximum value of the singularity rings of the LG-CCF (reached in far field) is $n+m$, that coincides with the conclusions in Refs. [6-8]. Analogously the maximum of the horizontal and vertical CCF singularity lines for the HG-SMB is $2 n$ and $2 m$ respectively as it is easy to conclude from the separability of the sums in Eq. (14) for the case of the HG-SMB. It seems that in far field for the relatively large $\sigma$, when HLG-SMB intensity distribution is almost Gaussian and the structure of mode cannot be distinguished, the singularity curves might serve as a fingerprint of the associated coherent mode.

Finally, in order to find the expression for the MI propagation in general rotationally symmetric system the following substitutions $\cos \alpha=a / \sqrt{a^{2}+b^{2}}$ and $\sin \alpha=b / \sqrt{a^{2}+b^{2}}$ in Eq. (15) together with addition of scaling and phase factor (see Eq. (5)) have to be done. Doing this we come to the following equation for the evolution of the MI of the HLG-SMB during 
(a)
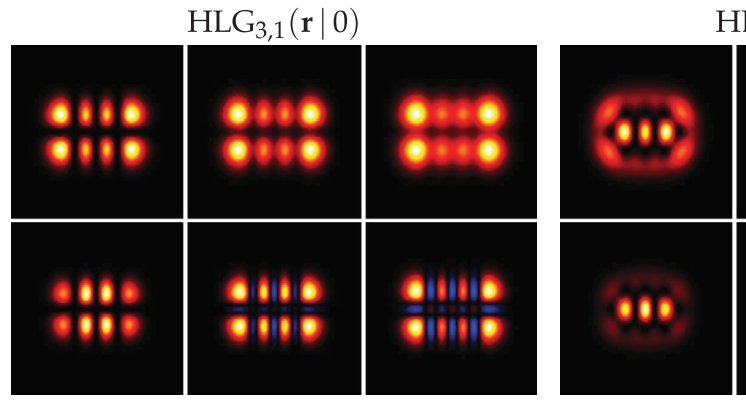

$\mathrm{HLG}_{3,1}(\mathbf{r} \mid \pi / 8)$

$\mathrm{HLG}_{3,1}(\mathbf{r} \mid \pi / 4)$
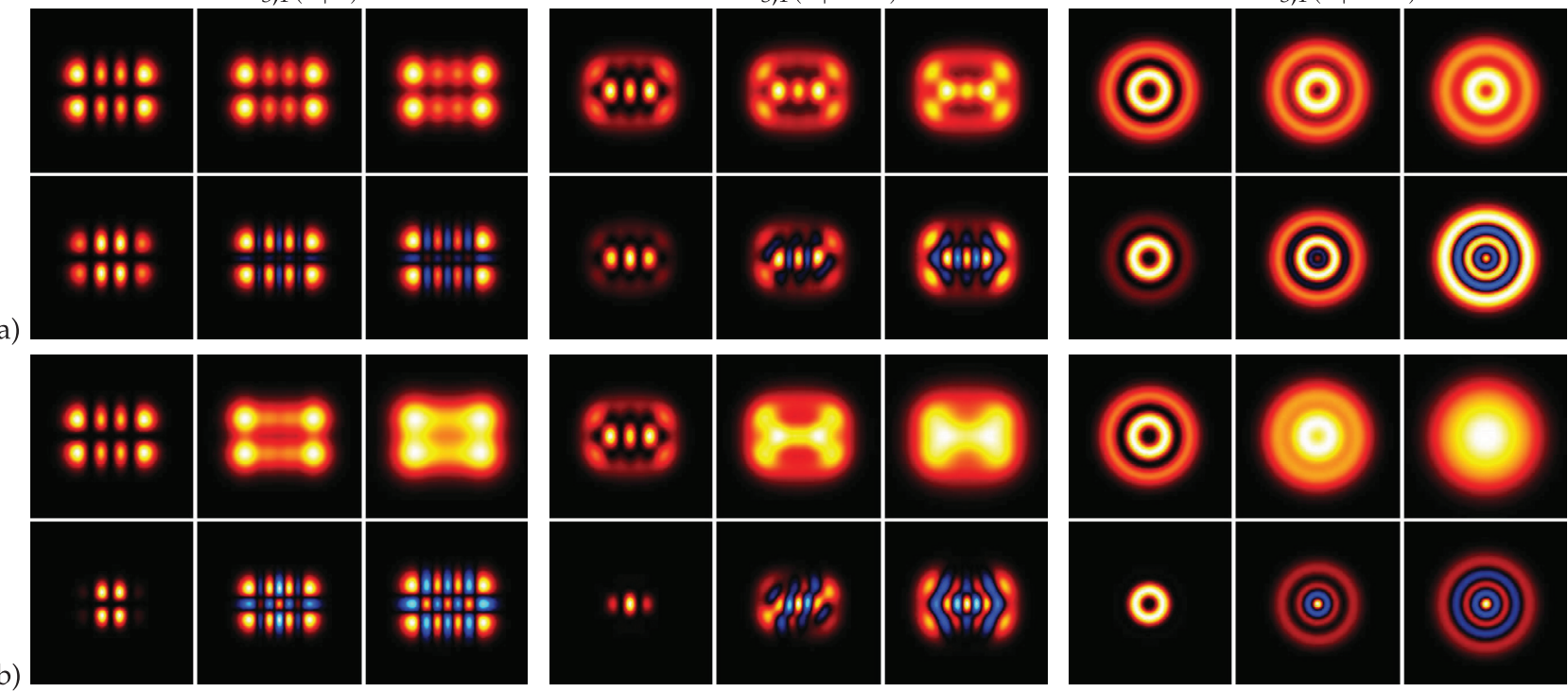

(b)
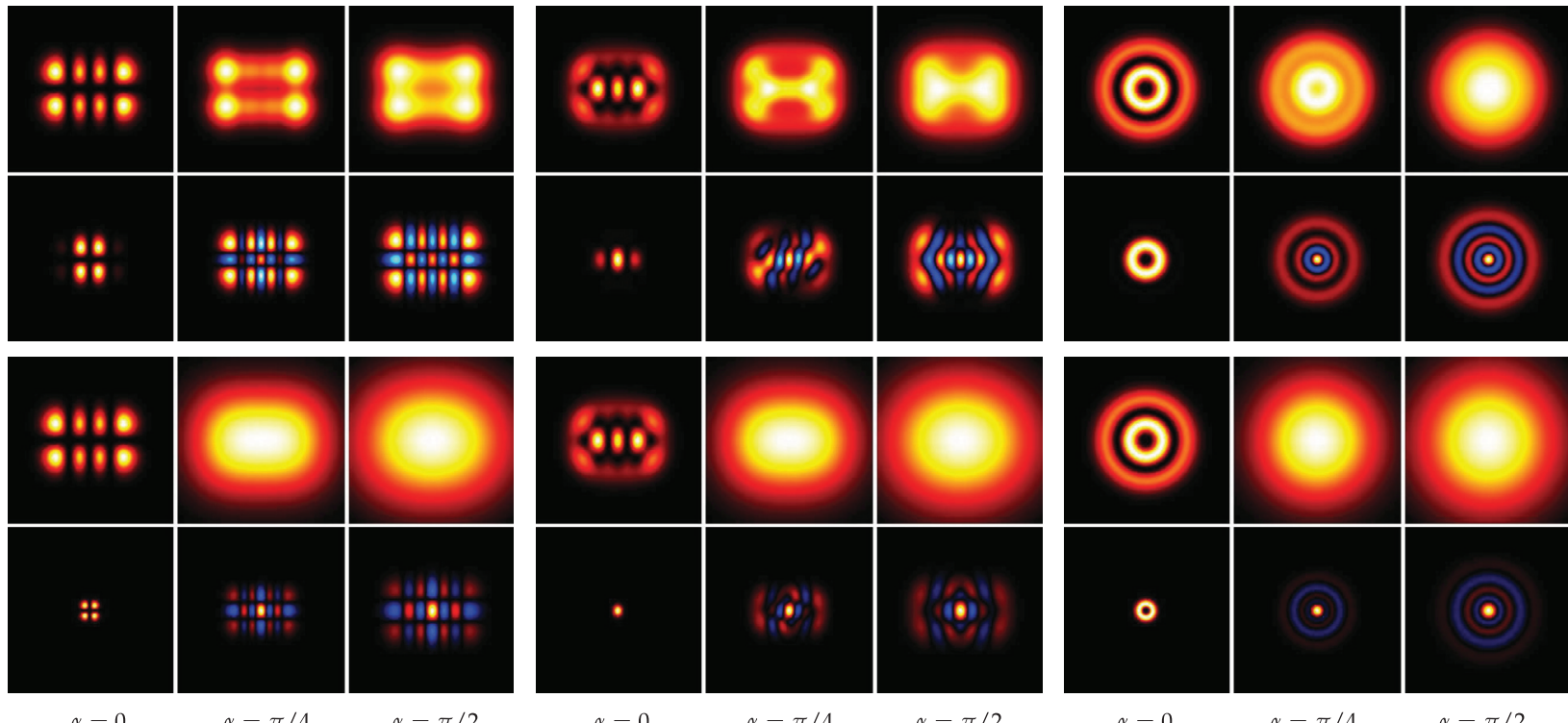

(c)

$$
\alpha=0
$$$$
\alpha=\pi / 4
$$

$$
\alpha=\pi / 2
$$

$\alpha=0$

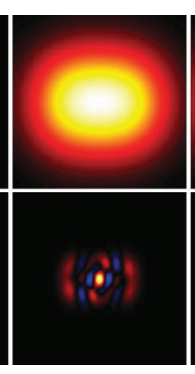

$\alpha=\pi / 4$
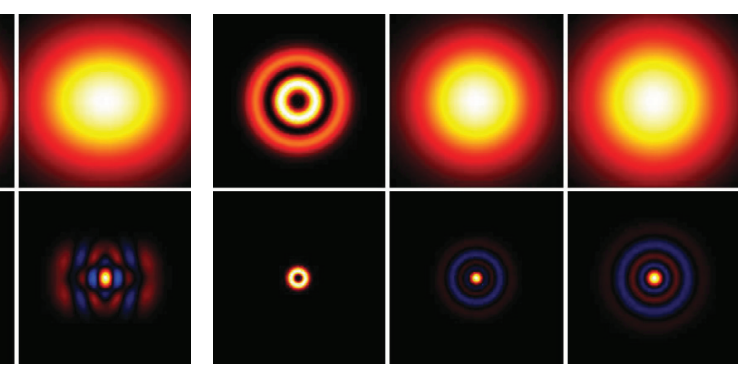

0

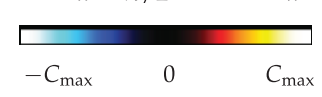

$\alpha=\pi / 2$

Fig. 1. Distributions of intensity and CCF of the HLG-SMB, $\operatorname{HLG}_{3,1}(\mathbf{r} \mid \beta)$, for $\beta=0, \pi / 8$, and $\pi / 4$ in the planes corresponding to $\alpha=0, \pi / 4$, and $\pi / 2$ for $\sigma=0.25$ (a), $\sigma=1(\mathrm{~b})$, and $\sigma=10$ (c). All frames are shown in a square $[-2,2] \times[-2,2]$.

propagation through the rotationally symmetric system:

$$
\begin{aligned}
& \Gamma_{n, m, \beta}^{(\sigma)}\left(\mathbf{R}+\frac{\mathbf{r}}{2}, \mathbf{R}-\frac{\mathbf{r}}{2} \mid \mathbf{T}\right)=\frac{1}{\left(a^{2}+[1+\sigma] b^{2}\right)^{n+m+1}} \\
& \times \exp \left(i 2 \pi \frac{a c+b d}{a^{2}+b^{2}} \mathbf{R}^{t} \mathbf{r}-\frac{\pi \sigma\left(a^{2}-b^{2}\right)}{2\left(a^{2}+b^{2}\right)^{2}}|\mathbf{r}|^{2}\right) \\
& \times \sum_{v=0}^{n} \sum_{\mu=0}^{m}\left(\begin{array}{l}
n \\
v
\end{array}\right)\left(\begin{array}{l}
m \\
\mu
\end{array}\right)\left(\sigma b^{2}\right)^{n+m-v-\mu}\left(a^{2}+b^{2}\right)^{v+\mu} \\
& \times \Gamma_{v, \mu, \beta}^{(0)}\left(\frac{\mathbf{R}+\frac{1}{2} q \mathbf{r}}{\sqrt{a^{2}+[1+\sigma] b^{2}}}, \frac{\mathbf{R}-\frac{1}{2} q \mathbf{r}}{\sqrt{a^{2}+[1+\sigma] b^{2}}} \mid \mathbf{T}_{0}\right)
\end{aligned}
$$

with $q=1+b \sigma(b-i a) /\left(a^{2}+b^{2}\right)$. Apart from the fundamental value of the comprehensive closed-form expression derived in this letter (see Eqs. (14), (15) and (19)) it is useful for beam analysis: identification of the mode associated with the HLG-SMB and its coherence degree. This finding might pave a way for the information encoding into partially coherent HLG beam.

Funding. Spanish Ministerio de Economía y Competitividad (TEC2014-57394-P).

\section{REFERENCES}

1. E. G. Abramochkin, T. Alieva, and J. Rodrigo, in Mathematical Optics: Classical, Quantum, and Computational Methods, (CRC, 2013), p.143.
2. M. J. Padgett, Opt. Express 25, 11265 (2017).

3. M. Chen, K. Dholakia, and M. Mazilu, Sci. Rep. 6, 22821 (2016).

4. G. Gbur, J. Opt. Soc. Am. A 31, 2038 (2014).

5. M. Born and E. Wolf, Principles of Optics (Cambridge University, 2006).

6. I. D. Maleev, D. M. Palacios, A. S. Marathay, and G. A. Swartzlander, Jr., J. Opt. Soc. Am. B 21, 1895 (2004).

7. D. M. Palacios, I. D. Maleev, A. S. Marathay, and G. A. Swartzlander, Jr., Phys. Rev. Lett. 92, 143905 (2004).

8. Y. Yang, M. Chen, M. Mazilu, A. Mourka, Y.-D. Liu, and K. Dholakia, New J. Phys. 15, 113053 (2013).

9. J. A. Rodrigo and T. Alieva, Opt. Lett. 40, 3635 (2015).

10. T. Alieva, J. A. Rodrigo, A. Cámara, and E. Abramochkin, J. Opt. Soc. Am. A 30, 2237 (2013).

11. E. G. Abramochkin and V. G. Volostnikov, Phys. Wave Phenom. 14, 14 (2010).

12. Y. Cai and C. Chen, J. Opt. Soc. Am. A 24, 2394 (2007).

13. F. Wang, Y. Cai, and O. Korotkova, Opt. Express 17, 22366 (2009).

14. L. Mandel and E. Wolf, Optical Coherence and Quantum Optics (Cambridge University, 1995).

15. F. Gori, Opt. Commun. 34, 301 (1980).

16. T. Alieva and M. J. Bastiaans, Opt. Lett. 30, 1461 (2005).

17. M. A. Alonso, Adv. Opt. Photon. 3, 272 (2011).

18. R. Simon and G. S. Agarwal, Opt. Lett. 25, 1313 (2000).

19. F. Gori, M. Santarsiero, R. Borghi, and S. Vicalvi, J. Mod. Opt. 45, 539 (1998).

20. G. Gbur and T. D. Visser, Opt. Commun. 222, 117 (2003). 


\section{FULL REFERENCES}

1. E. Abramochkin, T. Alieva, and J. Rodrigo, "Solutions of Paraxial Equations and Families of Gaussian Beams," in Mathematical Optics: Classical, Quantum, and Computational Methods, Eds. V. Lakshminarayanan, M. L. Calvo, and T. Alieva, chapter 5, pp.143-192 (CRC Press, 2013).

2. M. J. Padgett, "Orbital angular momentum 25 years on," Opt. Express 25, 11265-11274 (2017).

3. M. Chen, K. Dholakia, and M. Mazilu, "Is there an optimal basis to maximise optical information transfer?," Sci. Rep. 6, 22821 (2016).

4. G. Gbur, "Partially coherent beam propagation in atmospheric turbulence," J. Opt. Soc. Am. A 31, 2038-2045 (2014).

5. M. Born and E. Wolf, Principles of Optics (Cambridge University, 2006).

6. I. D. Maleev, D. M. Palacios, A. S. Marathay, and G. A. Swartzlander, Jr., "Spatial correlation vortices in partially coherent light: theory," J. Opt. Soc. Am. B 21, 18951900 (2004).

7. D. M. Palacios, I. D. Maleev, A. S. Marathay, and G. A. Swartzlander, Jr., "Spatial correlation singularity of a vortex field," Phys. Rev. Lett. 92, 143905 (2004).

8. Y. Yang, M. Chen, M. Mazilu, A. Mourka, Y.-D. Liu, and K. Dholakia, "Effect of the radial and azimuthal mode indices of a partially coherent vortex field upon a spatial correlation singularity," New J. Phys. 15, 113053 (2013).

9. J. A. Rodrigo and T. Alieva, "Evolution of coherence singularities of Schell-model beams," Opt. Lett. 40, 3635-3638 (2015).

10. T. Alieva, J. A. Rodrigo, A. Cámara, and E. Abramochkin, "Partially coherent stable and spiral beams," J. Opt. Soc. Am. A 30, 2237-2243 (2013).

11. E. G. Abramochkin and V. G. Volostnikov, Generalized Hermite-Laguerre-Gauss beams, Physics of Wave Phenomena, 14, 14-22 (2010).

12. Y. Cai and C. Chen, "Paraxial propagation of a partially coherent Hermite-Gaussian beam through aligned and misaligned ABCD optical systems," J. Opt. Soc. Am. A 24, 2394-2401 (2007).

13. F. Wang, Y. Cai, and O. Korotkova, "Partially coherent standard and elegant Laguerre-Gaussian beams of all orders," Opt. Express 17, 22366-22379 (2009).

14. L. Mandel and E. Wolf, “Optical Coherence and Quantum Optics" (Cambridge University, 1995).

15. F. Gori, "Collett-Wolf sources and multimode lasers," Opt. Commun. 34, 301-305 (1980).

16. T. Alieva and M. J. Bastiaans, "Mode mapping in paraxial lossless optics," Opt. Lett. 30, 1461-1463 (2005).

17. M. A. Alonso, "Wigner functions in optics: describing beams as ray bundles and pulses as particle ensembles," Adv. Opt. Photon. 3, 272-365 (2011).
18. R. Simon and G. S. Agarwal, "Wigner representation of Laguerre-Gaussian beams," Opt. Lett. 25, 1313-1315 (2000).

19. F. Gori, M. Santarsiero, R. Borghi, and S. Vicalvi, "Partially coherent sources with helicoidal modes," J. Mod. Opt. 45, 539-554 (1998).

20. G. Gbur and T. D. Visser, "Coherence vortices in partially coherent beams," Opt. Commun. 222, 117-125 (2003). 